\title{
Optimization of Integrated Electro-Absorption Modulated Laser Structures for 100 Gbit/s Ethernet Using Electromagnetic Simulation
}

\author{
Johansen, Tom Keinicke; Kazmierski, Christophe; Jany, Christophe; Jiang, Chenhui; Krozer, Viktor
}

Published in:

IMOC 2007

Link to article, DOI:

10.1109/IMOC.2007.4404320

Publication date:

2007

Document Version

Publisher's PDF, also known as Version of record

Link back to DTU Orbit

Citation (APA):

Johansen, T. K., Kazmierski, C., Jany, C., Jiang, C., \& Krozer, V. (2007). Optimization of Integrated ElectroAbsorption Modulated Laser Structures for $100 \mathrm{Gbit} / \mathrm{s}$ Ethernet Using Electromagnetic Simulation. In IMOC 2007 IEEE. https://doi.org/10.1109/IMOC.2007.4404320

\section{General rights}

Copyright and moral rights for the publications made accessible in the public portal are retained by the authors and/or other copyright owners and it is a condition of accessing publications that users recognise and abide by the legal requirements associated with these rights.

- Users may download and print one copy of any publication from the public portal for the purpose of private study or research.

- You may not further distribute the material or use it for any profit-making activity or commercial gain

- You may freely distribute the URL identifying the publication in the public portal 


\title{
Optimization of Integrated Electro-Absorption Modulated Laser Structures for $100 \mathrm{Gbit} / \mathrm{s}$ Ethernet Using Electromagnetic Simulation
}

\author{
Tom Johansen*, Christophe Kazmierski ${ }^{\dagger}$, Christophe Jany ${ }^{\dagger}$, Chenhui Jiang*, and Viktor Krozer* \\ *Technical University of Denmark, Oersted-DTU, Section of Electromagnetic Systems, \\ 2800 Kgs. Lyngby, Denmark, Email: tkj@oersted.dtu.dk \\ †Alcatel-Thales III-V Lab, F-91461 Marcoussis Cedex, France
}

\begin{abstract}
In this paper three options for very-high bit rate integrated electro-absorption modulated laser (EML) structures are investigated using electromagnetic simulation. A physics based distributed equivalent circuit model taking the slowwave propagation characteristics of the modulation signal into account is proposed for the electro-absorption modulator (EAM) electrode arrangement. This model makes it possible to apply an EM/circuit co-simulation approach to estimate the electrical to optical transmission bandwidth for the integrated EML. It is shown that a transmission bandwidth of $70 \mathrm{GHz}$ seems feasible with the investigated EML structures if the driving impedance is reduced to $25 \Omega$. It is also shown that the influence of electromagnetic cross-talk between components can be neglected in the estimation of the transmission bandwidth even in the case of very short separation zones. Finally, a slight decrease in transmission bandwidth is observed for an EML structure without ground pillars.
\end{abstract}

\section{INTRODUCTION}

The projected increase in data traffic motivates the development of very high bit rate optoelectronic transceivers suitable for $100 \mathrm{Gbit} / \mathrm{s}$ Ethernet type applications. Recently, the European Commission has launched the GIBON project to focus on the technology that will be used in the future for the integration of the highest speed optoelectronic transducers with their driving electronics [1]. Guidelines for the realization of these very-high bit rate integrated devices will be given by supporting electromagnetic (EM) simulation activities.

The electro-absorption modulator (EAM) fabricated on InP is a widely used transmitter component in high bit rate optical communication systems [2]-[3]. For the intended bit-rate of $100 \mathrm{Gbit} / \mathrm{s}$ the EAM offers several advantages such as large absorption variations at low driving voltages, very small size, and mature integration with a laser. The electrical to optical transmission bandwidth for an EAM is approximately limited by the time-constant $C_{s}\left(Z_{o} / 2+R_{s}\right)$, where $Z_{o}$ is the driver impedance, $C_{s}$ the EAM junction capacitance, and $R_{s}$ the EAM series resistance [4]. To obtain a sufficiently large transmission bandwidth $(>70 \mathrm{GHz})$ driver impedances $<50 \Omega$ are investigated in the GIBON project. The EAM will be monolithic integrated with a laser on InP to form an electroabsorption modulated laser (EML) structure suited for 100 $\mathrm{Gbit} / \mathrm{s}$. It is the focus of this paper to describe an EM/circuit simulation approach to investigate the performance of integrated EML structures for $100 \mathrm{Gbit} / \mathrm{s}$ Ethernet applications.

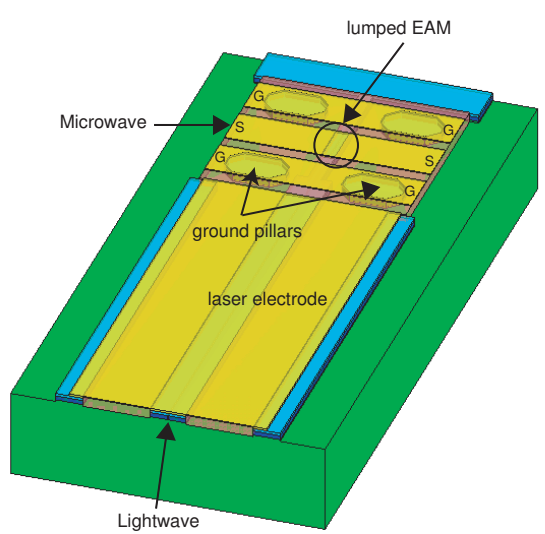

Fig. 1. 3D view of the integrated EML structure (option 1)

\section{INTEGRATED EML STRUCTURES}

A 3D view of the integrated EML structure proposed in the GIBON project is shown in Fig. 1. The length of the EML chip is $710 \mu \mathrm{m}$ including $220 \mu \mathrm{m}$ for the EAM part. To avoid any curves in the RF path an orthogonal microwave and lightwave propagation direction is proposed. A lumped EAM is included in the GSG electrode arrangement. The modulator length has been fixed at $50 \mu \mathrm{m}$ to give a good compromise between RC-parasitics and extinction ratio. A single active layer containing a $\mathrm{p}-\mathrm{i}-\mathrm{n}$ optical core is used for both the laser and the modulator part. The p-i-n optical core consists of an AlGaInAs multiquantum-well (MQW) stack. Electrical isolation between the two components are achieved using $\mathrm{H}+$ implantation. A heavily doped n-type InP substrate acts like the common ground electrode for the laser and modulator. A BCB polymer embedding the optical core reduces capacitance and provide isolation.

\section{A. GSG design options}

In this subsection the three different structures for the integrated EML investigated in this paper will be described.

1) Option 1: This structure is characterized by a $43 \mu \mathrm{m}$ distance between the laser end and beginning of the EAM. Ground pillars provide ground connection to the InP n-type substrate.

2) Option 2: The next structure is identical to the first structure except for a reduced distance between the laser end 


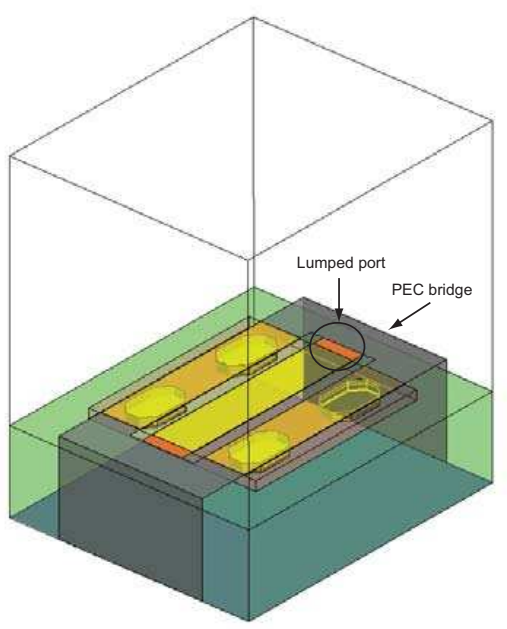

Fig. 2. Ansoft HFSS simulation setup for the EAM electrode arrangement

and the beginning of the EAM in order to lower the optical loss in the separation zone. Electromagnetic cross-talk is a concern with this structure and should be investigated.

3) Option 3: The final structure is identical to the first structure except the ground pillars are removed. This structure is investigated to check if the ground pillars are really necessary.

\section{EAM ELECTRODE ARRANGEMENT}

The starting point for our investigations has been to develope a distributed equivalent circuit model for the EAM GSG electrode arrangement. The setup in the commercial available simulator, Ansoft HFSS, is shown in Fig. 2. The coplanar excitation of the structure is obtained using lumped ports located across a gap between the signal line and a vertical PEC bridge which connects the two ground conductors together. The PEC bridge is wrapped around the edge to avoid problems with a floating substrate. This excitation resembles very well the measurement situation encountered using GSG probes but may also represent the excitation coming from the driver chip in the case a flip-chip approach. Unfortunately, a parasitic inductance and capacitance are associated with this excitation scheme. These parasitic elements must be evaluated and removed from the EM simulation results to obtain an accurate model of the electrode arrangement. Details in our parasitic removal method applied to EM simulation results can be found in [5]. The simulation structure is surrounded by an air box possessing the radiation boundary condition.

\section{A. EAM Electrode Modeling}

The Au electrodes are separated from the heavily doped InP substrate by a thin layer of $\mathrm{BCB}$ and thus forms a metal-insulator-semiconductor structure. It is well-known that this transmission line structure supports the slow-wave mode where the electrical field is concentrated in the isolating layer

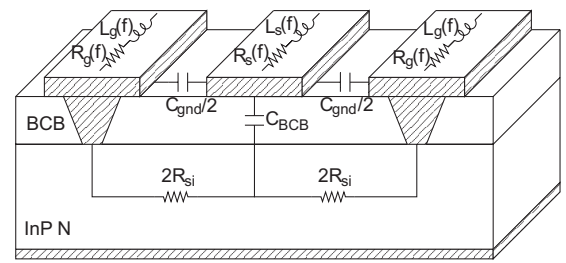

a)

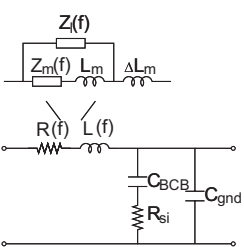

b)

Fig. 3. a) A section of EAM electrodes illustrating circuit elements. b) Elements of distributed equivalent circuit model.

beneath the center conductor while the magnetic field freely penetrates into the substrate [6]. This separation between the electric and magnetic field gives rise to the low-loss slow-wave mode of propagation. EM simulations performed on the EAM electrode arrangement verifies the slow-wave propagation mode even at frequencies up to $110 \mathrm{GHz}$ with an associated low attenuation of $\approx 2 d B / \mathrm{mm}$.

Fig. 3a) shows a section of the EAM electrodes. The elements of the distributed equivalent circuit model in Fig. 3b) follow from the physical structure in Fig. 3a). An inductance $L_{m}$ is associated with the longitudinal current which flows on the conductors. Induced Eddy-currents in the substrate are in parallel with this current flow. The effect of the induced Eddy-currents can be represented in the distributed equivalent circuit model as a parallel impedance $Z_{l}(f)$ calculated as

$$
Z_{l}(f) \approx \alpha(1+j) /\left(\delta_{s} \sigma_{s} W\right)
$$

where $\delta_{s}$ is the substrate skin depth, $f$ is the frequency, $\sigma_{s}$ is the conductivity of the substrate, and $W$ is the width of the center conductor. The above impedance can be deduced from the magnetic vector potential considering a single current sheet of width $W$ located above the substrate. The pre-factor $\alpha$ is a correction factor to take into account the actual geometry of the EAM electrode arrangement. The additional inductance $\Delta L_{m}$ serves the same purpose. To take the metallic conductive losses into account an impedance $Z_{m}(f)$ is introduced in series with $L_{m}$. This impedance is given by

$$
Z_{m}(f) \approx(1+j) /\left(\delta_{m} \sigma_{m} W\right)
$$

where $\delta_{m}$ and $\sigma_{m}$ are the metal skin depth and conductivity of the metal, respectively. The capacitance $C_{B C B}$ and resistance $R_{s i}$ are associated with the transverse electric field confined to the dielectric layer underneath the center conductor. There is also a capacitance $C_{g n d}$ associated with the transverse electric field in the air region. 


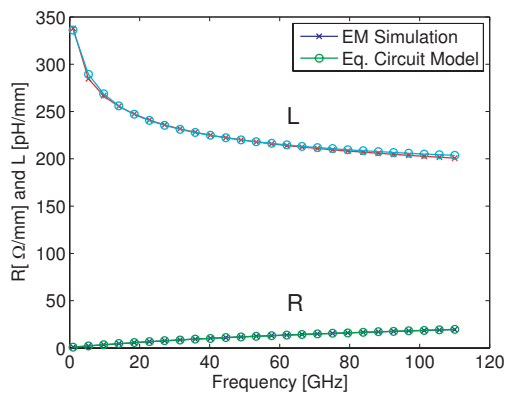

a)

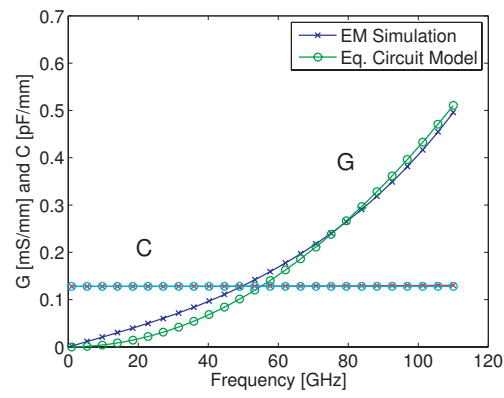

b)

Fig. 4. Comparison of EM simulated and modeled RLGC parameters for EAM electrodes.

In Fig. 4 the per-unit-length RLGC transmission line parameters extracted from the EM simulation results of the EAM electrode arrangement are compared with the developed model in Fig. 3b). It is observed that the developed model very accurately capture the dispersive behaviour of the per-unitlength RLGC parameters up to a frequency of $110 \mathrm{GHz}$.

\section{EM/CirCUit CO-Simulation APPROACH}

\section{A. Equivalent circuit model for EML structure}

To investigate the EML bandwidth potential of the EML structure the equivalent circuit model shown in Fig. 5 is purposed. The model has been implemented into the Agilent ADS microwave circuit simulator allowing both smallsignal AC responses and S-parameters to be easily calculated. Distributed transmission line sessions are included at the signal generator and load ends to take the slow-wave mode propagation characteristic of the modulating signal on the electrodes into account. The EAM part can often be modeled using a series resistance $R_{s}$ and capacitance $C_{s}$, and a dynamic photocurrent-resistance $R_{p h}$ [7]. The dynamic photocurrent resistance must be included to predict the effect of the optical power on the electrical-to-optical $(\mathrm{E} / \mathrm{O})$ response. The $\mathrm{E} / \mathrm{O}$ response is determined by the ratio of the voltage over the EAM diode junction $V_{\text {out }}$ to the voltage $V_{\text {in }} / 2$ applied to the EML structure in the case of zero input reflection. A shunt capacitor $C_{p}$ depending on the i-layer thickness in the region

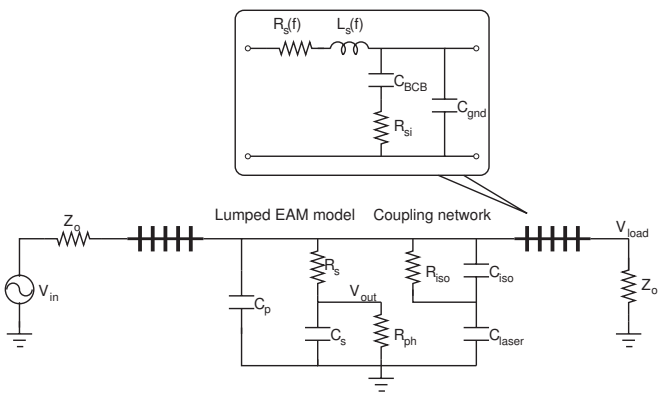

Fig. 5. Equivalent circuit model for EML bandwidth estimation. Insert shows distributed equivalent circuit model for electrode arrangement.

outside the EAM MQW stack is also included. To take the coupling to the laser part into account the isolation resistance $R_{i s o}$ and capacitance $C_{i s o}$ are used. The laser part itself is modeled here with a single capacitor $C_{\text {laser }}$.

\section{B. EM simulation setup}

It should be noticed that the lumped equivalent circuit model for the EAM diode junction depends on the optical power and applied signal swing in a nonlinear manner, and hence can not be predicted by EM simulation. Previously, EM simulation of EAMs has been performed by substitution of the MQW stack in the EAM diode junction with a dielectric material having an averaged permittivity [8]. In this work a slightly different approach is followed in that the lumped equivalent circuits for the MQW stack in the EAM and laser junction parts are included into the Ansoft HFSS EM simulation. This can be accomplished by using lumped RCL-boundary conditions available in the Ansoft HFSS simulator. An expanded view of the details in the EAM region shown in Fig. 6 illustrates the setup in Ansoft HFSS. For the EAM junction typical values for the capacitance $C_{s}$ and dynamic photocurrentresistance $R_{p h}$ are substituted, while for the laser MQW a

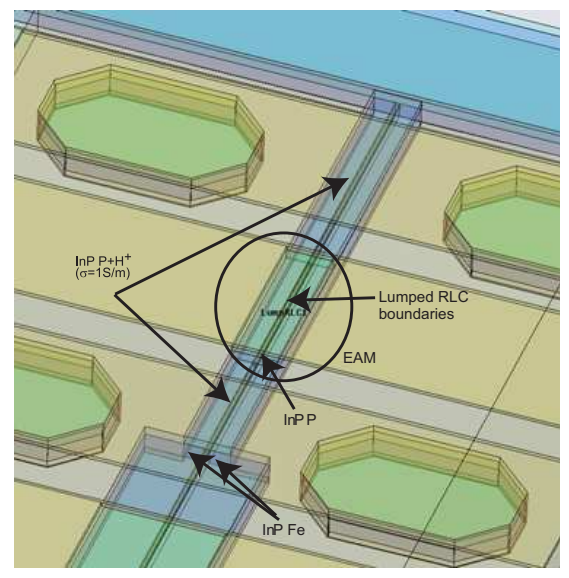

Fig. 6. Expanded view showing EAM region details for Ansoft HFSS integrated EML simulation setup. 


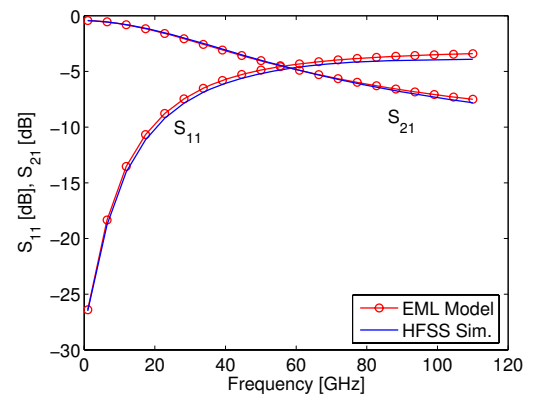

Fig. 7. Comparison of EM simulated and modeled electrical reflection and transmission responses for the EML structure (option 1).

single capacitor $C_{\text {laser }}$ is substituted. The junction capacitance values can be determined experimentally from low-frequency $\mathrm{C}-\mathrm{V}$ measurements, while the photocurrent resistance can be either calculated or measured with an optical input power to the EAM. In this way it is possible to obtain a more realistic view of the optical and electrical properties of the full EML structure from the EM simulation. The EM simulation results contain all the electromagnetic information of the EML structure, such as the electrical-to-electrical (E/E) transmission, port reflections, and coupling to the laser part. The output voltage $V_{\text {out }}$ across the EAM junction, however, which determines the $\mathrm{E} / \mathrm{O}$ response is not readily available from the EM simulation result. Therefore our approach is to fit the remaining elements $\left(R_{s}, C_{p}, C_{i s o}\right.$, and $R_{i s o}$ ) of the equivalent circuit model in Fig. 5 to the EM simulation results. An AC simulation can then be used to determined the E/O transmission bandwidth including the effect of driver impedances $Z_{o}<50 \Omega$.

The equivalent circuit modeling approach is verified in Fig. 7 by comparison of the EM simulated and modeled electrical reflection and transmission responses for the EML structure. It should be mentioned that the cross-coupling network has a very small influence on the simulated response. Especially it is difficult to extract a reliable value of the isolation resistance $R_{i s o}$ from the EM simulation result. To obtain a better estimate this resistance is calculated based on geometrical details.

\section{EML BANDWIDTH ESTIMATIONS}

In this section the $\mathrm{E} / \mathrm{O}$ transmission bandwidth for the three different structures for the integrated EML (option 1-3) described in section II is investigated. In order to investigate potential problems with electromagnetic cross-talk, option 2 , with reduced separation distance between the laser end and the beginning of the EAM is simulated in Ansoft HFSS. The obtained EM simulation result can not be distinguished from those of option 1 . The reason for this is that even with the shorter separation zone the calculated isolation resistance will still be on the order of hundreds of kilo-ohms due to the $\mathrm{H}+$ implantation. The electromagnetic cross-talk between components can therefore be neglected in the estimation of the transmission bandwidth as clearly observed in Fig. 8.

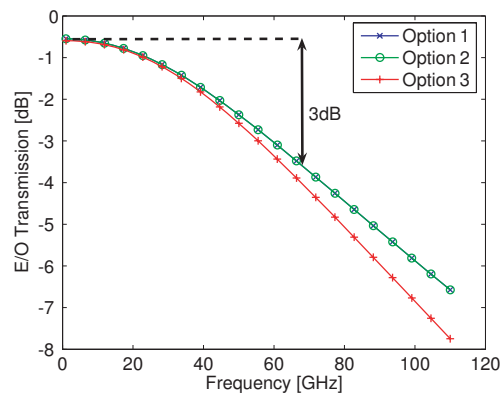

Fig. 8. Electrical to optical transmission characteristics for EML structures. The driver impedance level is reduced to $Z_{0}=25 \Omega$.

Notice that an E/O transmission bandwidth of nearly $70 \mathrm{GHz}$ is obtained when the driver impedance level is reduced to $Z_{o}=25 \Omega$. Even larger bandwidths are possible with further reduction of the driver impedance. To investigate if the ground pillars are really necessary, option 3, with ground pillars removed is simulated in Ansoft HFSS. The explanation of the slightly lower E/O transmission bandwidth observed in Fig. 8 is as follows. Without proper connection of the ground conductors to the heavily doped InP substrate a microstriplike mode is allowed to propagate along the GSG electrode. As a consequence the propagation characteristics of the GSG electrode changes and less of the supplied electromagnetic energy reaches the EAM diode junction. Instead of repeating the distributed equivalent circuit modeling for the GSG electrode without ground pillars the EAM part is substituted with an additional lumped port in the Ansoft HFSS setup. The lumped EAM model previously determined for the EML structure, option 1, is attached to this additional port in the Agilent ADS simulation.

\section{CONCLUSION}

In this paper three different EML structures for $100 \mathrm{Gbit} / \mathrm{s}$ have been investigated using EM simulation. Development of a distributed equivalent circuit model for the GSG electrode arrangement makes an EM/circuit simulation approach for determining the E/O transmission response possible. Employing a reduced driver impedance of $25 \Omega$ in connection with the integrated EML structures estimates an E/O transmission bandwidth of nearly $70 \mathrm{GHz}$. The influence of electromagnetic cross-talk on the bandwidth was shown to be negligible even in the case of very short separation zones. The transmission bandwidth for EML structure without ground pillars demonstrate slightly lower bandwidth than the two other structures investigated. Measurements performed on EML chips should be available at the time of the conference.

\section{ACKNOWLEDGMENT}

The authors would like to thank the European Commission for support under the 6th framework programme to the project 'Opto-electronic integration for 100 Gigabit Ethernet Optical Networks (GIBON)'. 


\section{REFERENCES}

[1] The GIBON website. [Online]. Available: http://www.istgibon.eu/index.htm

[2] M. L. Pallec et al., " $42 \mathrm{GHz}$ bandwidth InGaAlAs/InP electro absorption modulator with a sub-volt modulation drive capability in a $50 \mathrm{~nm}$ spectral range," in Proc. IPRM 04, 2004.

[3] H. Fukano et al., "Very-low-driving-voltage electroabsorption modulators operating at $40 \mathrm{~Gb} / \mathrm{s}$," J. Lightwave Technol., vol. 24, no. 5, pp. 22192224, 2006

[4] H. Arimoto et al., "A 40-Gbit/s electro-absorption modulator with a record modulation efficiency $(50 \mathrm{GHz} / \mathrm{V})$ enhanced by a novel technique hybrid integration on the driver IC," in Proc. LEOS 03, 2003, pp. 646-647.

[5] T. K. Johansen et al., "Em simulation accuracy enhancement for broadband modeling of on-wafer passive components," in To be published Proc. EuMIC 07, 2007.

[6] Y. R. Kwon et al., "Quasi-TEM analysis of slow-wave mode propagation on coplanar microstructure MIS transmission lines," IEEE Trans. Microwave Theory Tech., vol. MTT-35, no. 6, pp. 545-551, 1987.

[7] G. L. Li et al., "Concise RF equivalent circuit model for electroabsorption modulators," Electron. Lett., vol. 36, no. 9, pp. 818-820, 2000

[8] T. Yamanaka et al., "Lightwave-microwave unified analysis of electroabsorption modulators integrated with RF coplanar waveguides," IEEE Photon. Technol. Lett., vol. 17, no. 12, pp. 2562-2564, 2005. 